\title{
Nemorubicin Hydrochloride
}

National Cancer Institute

\section{Source}

National Cancer Institute. Nemorubicin Hydrochloride. NCI Thesaurus. Code C84000.

The hydrochloride salt form of nemorubicin, a morpholinyl analogue of the anthracycline doxorubicin with antineoplastic activity. Nemorubicin is metabolized via the P450 CYP3A enzyme to a highly cytotoxic derivative. Unlike most anthracyclines, nemorubicin is a topoisomerase I inhibitor and appears to exert its effect through the nucleotide excision repair (NER) system. In addition, this agent does not show cross-resistance with other anthracyclines. 